\title{
A FORMAÇÃO DO NEUROLOGISTA PARA O SECULO XXI
}

\author{
MARCO AURELIO LANA-PEIXOTO :
}

\begin{abstract}
RESUMO - A formação do neurologista inclui a aquisição de amplo conhecimento de medicina interna, das neurociências básicas e das doenças do sistema nervoso. Além de obter conhecimento cientifico o neurologista deve desenvolver habilidades clínicas a atitudes que permitam a expressão adequada de sua competência e seu contínuo aperfeiçoamento profissional. Para atingir este objetivo ha necessidade de promover profundas mudanças no sistema de educiação médica inserindo-o no espírito universitário da pesquisa e da busca da excelência. A residência médica deve enfatizar a formação de hábitos de aprendizado independente, do uso da metodologia científica, de pensamento analítico e crítico, assim como de atitudes éticas. Neste sentido o preceptor desempenha importante papel como modelo.
\end{abstract}

PALAVRAS-CHAVE: educacăo médica, neurologista, residente, preceptor, metodologia científica.

The education of neurologists for the 21st century.

SUMMARY - The training of neurologists for the practice in the coming years requires deep changes in the system of medical education. It is critical for the medical student and the resident the acquisition of a broad knowledge of scientific information. But physicians must also know how to use knowledge they have accumulated from a variety of sources. They express their knowledge through abilities in the wards or in ambulatory gettings in order to get an accurate diagnosis and the proper management of a particular condition. In addition to that they are expected to develop ethical attitudes toward their patients, peers and the greater society. They must be committed to work, to independent learning and to rationality. To accomplish its scholarly goals medical education has to emphasize the development of adequate personal attributes, skills and habits of mind rather than the transmission of factual information. With todiay's technology all the soientific information necessary for the practice of medicine is at the fingertips through computers. Memorization is therefore an absolute nonsense in teaching. A better education involves reducing the working load and the number of lecture hours providing students and residents with sufficient unscheduled time to seek out information independently and to assess their own learning. Problem-solving methods of teaching providing the formulation of multiple concurrent hypotheses, the critical analysis and evaluation of data and the application of new acquired knowledge as well as previously learned concepts to the solution of problems should be widely employed. Faculty members are guides who assist studentsi and residents to develop analytical and critical skills. They play an important role as models through their own attitudes and actions. The humanistic dimensions of the medical profession cannot be overemphasized.

KWY WORDS: medical education, neurologist, resident, house-staff, role models, scientific methods.

A revolução biomédica das últimas décadas trouxe enorme progresso das ciências neurológicas. O sistema de ensino médico, no entanto, parece ineficaz em capacitar especialistas para exercer a neurologia de acordo com os recentes avanços tecnológicos incorporados aos princípios e objetivos primordiais da medicina 15.

* Professor Adjunto de Neurologia da Faculdade de Medicina da Universidade Federal de Minas Gerais (UFMG). Aceite: 26-janeiro-1993.

Dr. Marco Aurélio Lana-Peixoto - Instituto Hilton Rocha - Av. Anel da Serra 1355 - 30210-090 Belo Horizonte MG - Brasil. 
Este artigo discute os traços fundamentais que devem caracterizar o neu. rologista e destaca algumas medidas educacionais necessárias para seu desenvolvimento e aprimoramento a fim de que ele possa desempenhar suas funções de acordo com os padrões científicos de sua era e os preceitos éticos e morais permanentes da profissão.

\section{AS QUESTOES PRELIMINARES}

Quando se considera a formação de neurologistas para os próximos anos, coincidentemente, a virada para um novo século, algumas questões necessariamente se interpõem: (1) 0 que é um neurologista e o que ele faz?; (2) que bagagem de conhecimentos ele deve adquirir para exercer suas funções profissionais eficientemente,; (3) que habilidades deve ele desenvolver para desempenhar suas funções usando os conhecimentos disponiveis?; (4) que atitudes deve expressar em relaçăo a seus pacientes, a seus colegas e à sociedade?; e por fim (5) que atitudes pessoais deve desenvolver em relacão a si próprio, à necessidade de seu contínuo aperfeiçoamento, ao fenômeno da vida, do sofrimento e da mortê?

Pode-se dizer que o neurologista ê um médico que possui um conjunto de conhecimentos cientificos, de habilidades clínicas e de atitudes que vão lhe capacitar para o diagnóstico e o tratamento das doenças do sistema nervoso, assim como para o cuidado e o bem-estar de seus pacientes. Toda a prática médica se assenta sobre estes três pilares: o conhecimento científico, as habilidades clínicas $e$ as atitudes. O conhecimento científico sem as habilidades clinicas para expressá-lo e as atitudes para humanizá-lo torna-se absolutamente inútil.

o conhecimento cientifico. O neurologista deve ter amplo conhecimento de medicina geral $e$ profundo conhecimento das neurociências e das desordens do sistema nervoso. $O$ pré-requisito de um ano de medicina interna para a residência em neurologia é importante para consolidar os conhecimentos de clínica médica o preencher lacunas frequentes no curso de graduação.

Um profundo conhecimento das ciências neurológicas básicas é também fundamental para o exercício diário da neurologia. A compreensão daś manifestações das doenças do sistema nervoso só será possivel através de sólido conhecimento científico de anatomia, físiologia, bioquimica e patologia. O aprendizado então se fará através das associações mentais entre os fenómenos observados e os conceitos adquiridos das ciências básicas.

Por fim o neurologista deve possuir amplo e profundo conhecimento da história natural, dos sintomas e sinais das doenças que afetam o sistema nervoso, acompanhando rigorosamente o progresso no desvendamento das causas e mecanismos fisiopatológicos, assim como o desenvolvimento dos métodos diagnósticos e de tratamento.

As habilidades clinicas. As habilidades clinicas são os mecanismos através dos quais os conhecimentos cientifcios são empregados no contato com os pacientes, na identificação das manifestaçōes das doenças, na formulação das hipóteses diagnósticas e na proposição do tratamento. Há necessidade de habilidades bem definidas para a realização da anamnese e do exame físico, para a seleção e interpretação de exames complementares, para o desenvolvimento do raciocínio diagnóstico e para a instituição da terapêutica adequada.

Todo este processo da aplicacão do conhecimento científico para resolver um problema, diante da situação real de um paciente, é chamado competência/8. O conhecimento cientifico é o saber, a habilidade clinica na aplicação do conhecimento, ou sejla, o saber como é a competência. A competência, portanto se apoia no conhecimento a se expressa na praxis, no atendimento aos pacientes nos ambulatórios e nias enfermarias.

As atitudes. O neurologista necessita, para se comportar como agente da relação entre o conhecimento científico e o doente, de desenvolver determinadas atitudes pessoais e interpessoais com seus pacientes e familiares, com os coleglas, as entidades médicas e toda a sociedade.

$O$ ato médico não depende apenas de coleção de conhecimentos científicos, que no mundo moderno, são extremamente instáveis, a todo momento substituídos por «novas verdades». Tamberm ele năo se realiza, totalmente ainda, pela competência, no emprego adequado do conhecimento vigente através das habilidades clínicas. $O$ ato médico apenas se completa na vida de relaçăo do médico com seu paciente (Fig. 1).

O médico não se relaciona com a doença como entidade abstrata e isolada, mas com uma pessaa portadora de uma doença. Portanto, o ato médico consiste nas relações do conhecimento cientifico com a realidade existencial de uma pessoa doente. $O$ médico é o agente 
desta relação. Através de seus conhecimentos, de suas habilidades, do raciocínío lóglco e do julgamento clínico, ele identifica a doença e estabelece o tratamento.

Como agente intermediário entre a ciência e o doente, o médico humaniza a relação do fato cientifico vigente - sempre imperfeito, fragmentado e instável - com a realidade existencial da dor, da incapacidade e do sofrimento. Ao agir sobre estia realidade e ao modificá-la, ele se depara com a oportunidade, impar entre todas as profissóes, de também se modificar, desenvolvendo sua sensibilidade a.os valores objetivos e imutáveis da vida (Fig. 2).

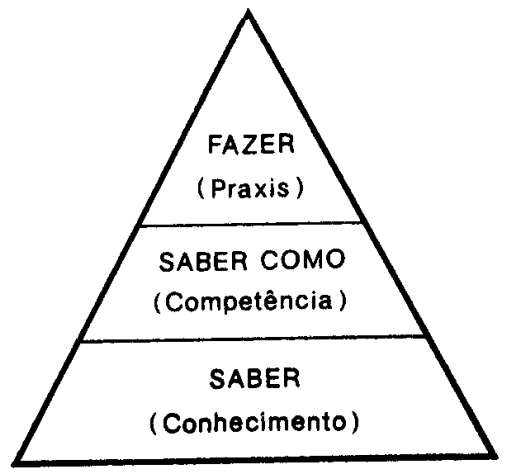

Fig. 1. A piramide do ato médico.

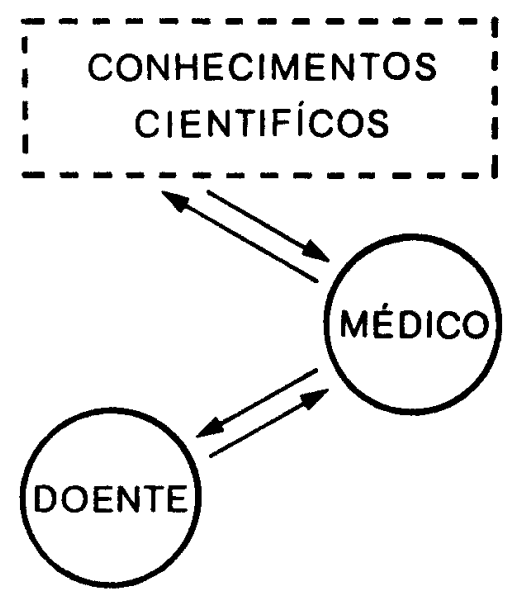

Fig. 2. As relações do ato médico.

O neurologista necessita ainda desenvolver atitudes pessoais que proporcionem a continua busca do saber e do aperfeiçoamento de suas habilidades. Wle deve expressar atitudes que revelem seu profundo compromisso com o trabalho, o serviço a seus pacientes e a sociedade, assim como aguçar seu espirito autocritico no constante questionamento de seus atosib.

\section{A FORMACAO dO NEUROLOGISTA}

Uma vez delineadas as características básicas do neurologista, devemos considerar os meios necessários a sua formação e desenvolvimento. Uma visão ampla da inserção da medicina no mundo moderno é útil na discussão de medidas educacionais.

O ambiente científico do século XX. Presenciamos neste final de século uma grande revolução biomédica em que os mecanismos mais íntimos das doenças, em nível molecular vão sendo desvendados. Há poucos anos atrás nāo imaginávamos que tão cedo entenderíamos a patogênese molecular da distrofia muscular de Duchenne ou da neuropatia óptica hereditária de Leber. As grandes conquistas biológicas de hoje têm sido comparadas aos grandes marcos da história da humanidade, tais como a pintura do Renascimento, à música do século XVIII e à física quântica do século XX 15. Estamos hoje no limiar do conhecimento de como os gens atuam influenciando os caracteres humanos - desde os seus traços físicos aos dotes intelectuais, desde como determinam a inteligência até como préestabelecem a ocorrência das doenças e a própria extensão da vida.

O século XX termina em grande ebulição cientffica, nunca sonhada. Não só os mistérios das causas das doenças vão sendo revelados mas os métodos de diagnóstico permitem avaliações bioquímicas e imunológicas precisas assim como imagens, quase perfeitas, de órgãos e sistemas em pleno funcionamento.

O desenvolvimento da computação atinge profundamente o ambiente médico 1,17 . Pesquisas bibliográficas, antes exaustivas e demoradas, são agora exequiveis em poucos minutos; 100000 páginas de texto são colocadas em apenas um pequeno disco e todas as informações nelas contidas tornam-se repidamente 
disponiveis pelo uso de algumas palavras-chave. Relações de possíveis doenças com indicação de testes para a confirmação diagnóstica podem ser obtidas pelo fornecimento de dados da história e do exame físico de um determinado paciente. Tudo isto torna a memorização uma prática obsoleta.

As pressões econômicas e sociais. Nesta virada de século o exercício da medi. cina sofre enormes pressões econômicas e sociais que gradativamente moldam a prática médica. Nosso maior desafio é como assegurar que a medicina, e mais precisamente a neurologia, possa responder satisfatoriamente às pressões econômicas e sociais de nosso meio. A medicina deve incorporar os avanços científicos e tecnológicos do mundo moderno a seus valores objetivos e permanentes, adaptando sua prática às pressóes do aumento da demanda de serviços e da contenção de custos, resistindo bravamente porém à sua massificação e desumanização 4,21 .

Embora seja impossível prever toda a gama de influência que as próximas décadas irão trazer, o certo é que a neurologia mudará radicalmente. Temos que nos esforçar para que tenhamos participação ativa na remodelação da prática neurológica nos próximos anos, junto às forças econômicas e sociais sobre as quais não exercemos maior influência.

o primeiro passo: a reforma do ensino médico. Devemos iniciar uma grande reforma em nosso sistema educacional de formação de médicos e de neurologistas, adaptando-o às novas condições de desenvolvimento tecnológico e à realidade econômica e social do mundo moderno. Mesmo em países desenvolvidos esta mudança tornou-se necessária. O ensino médico nos Estados Unidos tem sido comparado aos dinossauros 24 que reinaram sobre a Terra por longo período, mas que desapareceram porque foram incapazes de se adaptar às novas condições ambientais. Assim, todo o sistema de ensino médico americano, que moldou o mundo em quase todo este século, estará ameaçado de extinção se profundas mudanças não forem promovidas, expandindo e implimentando em toda sua extensão e profundidade as premissas fundamentais do Relatório Flexner.

As mudanças do ensino médico visam a dois grandes objetivos: a excelência da educação profissional e da prática médica, e a formação de líderes com a visão necessária para continuamente transformar o que é naquilo que deve ser 24 . No Brasil o problema é ainda mais grave, pois sofremos com maior intensidade a pressão das questões econômicas e sociais.

O que necessitamos é colocar a educação médica dentro do espírito da universidade 23, ou seja do saber, do questionamento e da investigação científica. $A$ universidade do século $\mathrm{XX}$ deixou de ser o tranquilo centro de ortodoxia ou local de ensino de idéias estabelecidas para se transformar em centro de indagação e de crítica aos conceitos e valores dominantes, em local de dúvida e de busca, de aprimoramento e de pesquisa 21 . A universidade deixou de ser um centro de dogmas para se transformar em um centro de ceticismo, de novas idéias, livre de autoridades pré-determinadas. O dogmatismo que caracterizou Galeno e seus discípulos é um exemplo do perigo e da tirania da autoridade. A autoridade dos textos gregos se tornou uma barreira que impediu o progresso da medicina por muitos séculos 21 .

A visão de Flexner. Abraham Flexner surge como o grande mentor da educação médica deste século. Foi ele que inseriu a educação médica dentro do espírito universitário moderno da pesquisa científica e da procura da excelência. $O$ Relatório Flexner de 19108 estabeleceu que a educação médica deve se apoiar em solidas bases científicas combinadas com a prática clínica num ambiente universitário de pesquisa. Daí veio a grande revolução médica americana que determinou os padrões de ensino e de prática médica em todo o mundo ocidental neste século.

O equilíbrio entre o aprendizado da teoria e da prática já havia sido pre. conizado desde os dias de John Morgan, fundador da primeira escola de medicina do Novo Mundo 23. Morgan rompeu com o sistema tradicional de ensino, exclusivamente dentro do hospital, baseado em rígidas condutas dogmáticas. Ele preparou o caminho para Flexner a fim de que o academicismo médico florescesse dois séculos depois. 
No Brasil o desenvolvimento da medicina chega de maneira truncada e desordenada, desorientando ainda mais a educação médica. Daí a urgência da reforma de nosso sistema educacional. Precisamos atingir aqui a era americana de 1910. Como Flexner recomendou nos Estados Unidos, naquele tempo, necessitamos hoje no Brasil, também, fechar os cursos médicos de má qualidade, reduzir o número de alunos, atrelar o ensino clínico ao estudo das ciências básicas, desenvolver a pesquisa e contentar apenas com o padrão de excelência.

$O$ aprendizado médico deve sair das salas de aula e encontrar os pacientes nos ambulatórios e enfermarias 2,8 , sempre no entanto correlacionando os dados de história e de exame com os fundamentos científicos. O ensino nos ambulatórios deve ser priorizado 20, uma vez que: (1) a grande maioria dos pacientes pode ser tratada ambulatorialmente; (2) as internações tornaram se reservadas para os casos mais graves; (3) o tempo de permanência hospitalar é hoje reduzido por razóes econômicas, diminuindo as oportunidades de ensino; (4) muitas admissões são feitas apenas para procedimentos específicos; e (5) a maioria das decisōes é tomada nos ambulatórios.

O preceptor como modelo. Independentemente do ambiente de ensino, quer nos ambulatórios ou nas enfermarias, o preceptor é sempre um modelo para os alunos e médicos residentes. Nos laboratórios ou nas salas de aula o professor serve como modelo demonstrando domínio da matéria, pensamento analítico e se comportando com ética profissional. No ambulatório ou junto ao leito o preceptor é modelo ao demonstrar suas habilidades no contato com o paciente, durante o exame e ao tomar decisões amparadas no conhecimento cientifico e no raciocínio lógico. Em todas as situaçōes, no entanto, o preceptor deve expressar entusiasmo por seu trabalho, desejo de contínuo aprendizado, autoconfiança, responsabilidade, respeito pelos outros e por outras áreas profissionais e reconhecimento de suas próprias limitaçōes 7,11. Os traços dos preceptores que os alunos e residentes mais valorizam são o amplo conhecimento médico, o entusiasmo, a competência na solução dos problemas, a acessibilidade $e \quad 0$ interesse pelos alunos e pacientes. As características negativas mais frequentemente apontadas são a arrogância, o desgosto pelo ensino, a falta de autoconfiança, o dogmatismo, o desrespeito pelos estudantes e residentes e a insensibilidade aos problemas dos outros 10 .

A metodologia na residência. Quando nos questionamos a respeito do ensino na residência médica devemos nos lembrar que nem a medicina pode ser aprendida durante o curso médico ${ }^{5}$ nem a neurologia pode ser aprendida durante a residência. Não é interessante, portanto, sobrecarregar o aluno e o residente com um grande pêso de informações científicas $1,5,17,22$, que a todo momento são substituídas. $\$$ muito mais importante que sejam identificados os conceitos e princípios fundamentais sobre os quais a neurologia se apóia. Deve-se dar pouca ênfase à necessidade de memorização de informações. Nesta era de computadores a memorização tornou-se inútil 1,12,17. O aprendizado deve se deslocar para a formação de hábitos 13 , tanto de observação de fenômenos e da busca independente de informaçōes para explicá-los, quanto de atitudes na vida de relação. O professor é modelo e orientador no desenvolvimento destes hábitos.

A carga de trabalho e de obrigações formais não deve ser tão grande, a fim de que haja tempo adequado para o estudo independente, a busca de informações e para a análise crítica do próprio aprendizado 6,16,23. O neurologista deve estar preparado para aprender sozinho durante toda a sua vida 16, empregando os novos conhecimentos e habilidades na solução dos problemas diários.

A metodologia científica e a formação do «expert». $O$ estudante e o residente devem se habituar ao uso da metodologia cientifica na solução dos problemas de seus pacientes. O método científico ${ }^{3}$ se inicia pela observacão de um fenómeno e pela associação entre este estímulo externo e os conceitos previamente aprendidos. Esta etapa é a mais criativa em todo o processo e dela resultam as hipóteses diagnósticas. A segunda etapa é formulação de experimentos para verificar as hipoteses, ou seja, no caso do médico, é o exame físico ou a seleção de exames complementares. A terceira etapa consiste na coleta dos dados ou resultados, e a última etapa é a análise destes resultados, que levará a uma das três conclusões: (1) os dados confirmam a hipótese; (2) os dados afastam a hipótese; (3) os dados são inadequados ou insuficientes de modo que novos exames ou experimentos são necessários (Fig. 3). 
OBSERVACĀO DO

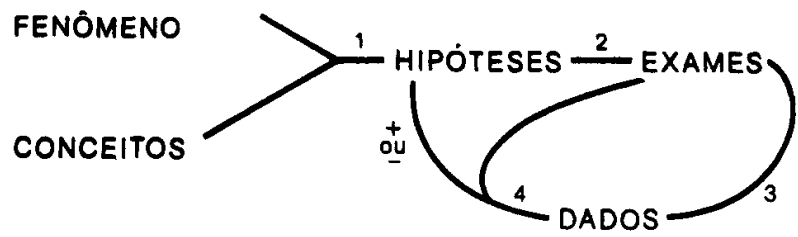

Fig. 3. As etapas do método científico. Modificado de Dreyfus \& Dreyfus, 1986.

A primeira etapa do método científico, ou seja a realização de associações entre o estímulo externo - como por exemplo a queixa principal do paciente e os conhecimentos anteriormente obtidos relacionados a este estímulo, é o ponto mais importante do aprendizado. E por isto que os fatos e conceitos devem ser ensinados no contexto dos estímulos externos, ou seja, diante da situação real de um paciente 12. A educação médica deve se processar, portanto, através das associações entre os fenômenos observados das doenças e os conceitos ou prin. cípios básicos, mesmo que isto implique ensinar menos conceitos 22 .

Para um médico experiente 19 a realização de um diagnóstico é apenas o processo de reconhecimento do "script» da doença, já existente em sua memória e adquirido de experiências anteriores. Quando confrontado a situação nova, no entanto, sobre a qual ele não tem experiência prévia, o médico usa o mesmo processo básico do método científico para chegar ao diagnóstico.

Para se tornar um "expert»19 o neurologista necessita percorrer algumas etapas transitórias. Inicialmente ele deve aprender as regras e então aplicá-las indistintamente, sem considerar a natureza da situação. A medida em que ele progride através da experiência real, ele vai modificando as regras dependendo das circunstâncias e acumulando representações mentais das doenças. Ele se tornará então um neurologista experiente quando tiver um grande número de representações mentais que serão chamadas à lembrança, de imediato, quando exposto a um caso semelhante. O diagnóstico será então fundamentalmente intuitivo, havendo supressão da primeira etapa do método científico, ou seja, da associaçã̃o da observação do fenômeno com os conceitos básicos. $O$ neurologista "expert", no entanto, mais que o experiente, além de usar as representações mentais acumuladas de experiências prévias no reconhecimento daquela situação específica e de chegar ao diagnóstico de maneira intuitiva 22 , ele é capaz de reconhecer e de explicar semelhanças e diferenças da situação presente com seus modelos mentais, a partir do exercício consciente de fazer associações entre os fenômenos observados e os conceitos básicos. Portanto mais que o médico experiente, o wexpert» tem visão crítica de sua própria intuição e através da associação observacão dos fenômenos - conceitos ele explica, modif̣ica e transcende o diagnóstico intuitivo e suas próprias representações mentais.

A prática neurológica deve ser, portanto, um exercício intelectual constante de associar as observaçōes dos fenômenos das doenças com os conhecimentos básicos e conceitos previamente aprendidos a fim de confirmar ou modificar o diagnóstico intuitivo. Deste exercício resultará o neurologista "expert», crítico e criativo.

A literatura médica. A maneira mais adequada para se obter novas informações e conceitos é o relacionamento regular e sistemático com a literatura médica 14,17. A residência deve incentivar o hábito de busca de informações através da leitura de trabalhos originais. Deve-se aprender não apenas selecionar os artigos originais relevantes para a prática mas, também, desenvolver a capacidade de leitura analítica e crítica, verificando a metodologia empregada e os resultados obtidos para depois correlacioná-los aos próprios conceitos e experiências.

A consciência humanistica. Considerados os requisitos técnicos e científicos, as habilidade e os hábitos mentais, a formação do neurologista ainda estará incompleta se não houver desenvolvimento da consciência de sua própria humanidade. Esta consciência se revela através de suas atitudes, principalmente na relação com seus pacientes. Nenhuma experiência humana se iguala à relação do médico 
com seu paciente 6 . Atitudes humanísticas são ensinađas principalmente através do exemplo dos preceptores 26 . A ética é então aprendida nas atitudes da vida quotidiana.

A tecnologia traz o risco da desumanização do encontro médico-paciente. Os estudantes tornam-se gradativamente menos altruistas à medida em que progridem no curso médico e tomam contato com equipamentos mais sofisticados ? Cabe aos preceptores reverter esta tendência, fazendo com que os alunos e residentes identifiquem em seus pacientes a sua própria natureza, seus mesmos anseios e seu igual destino.

\section{REFTRENCIAS}

1. Clawson DK. The education of the physician. Acad Med 1990, 65:84-88.

2. Dauphinee WD. Clinical education: the legacy of Osler revisited. Acad Med 1990, 65(Suppl) :68-73.

3. Dreyfus HL, Dreyfus SE. Mind over machines. New York: Free Press, 1986.

4. Eichna LW. Medical-school education, 1975-1979: a student perspective. N Engl J Med 1980, 303:727-734.

5. Elichna LW, A medical-school curriculum for the 1980s. N Engl J Med 1983, 308:18-21.

6. Federman DD. The education of medical students: sounds, alarms, and excursions. Acad Med 1990, 65:221-226.

7. Eicklin FL, Browne VK, Powell RC, Carter JE. Faculty and house stiaff members as role models. J Med Educ 1988, 63:392-396.

8. Flexner A. Medical education in the United States and Canada: a report to the Carnegit Foundation for the Advancement of Teaching. Bulletin N. 4. Boston, Massachusetts: Updyke, 1910.

9. Gorlin -R, Zucker HD. Physicians' reactions to patients: a key to teaching humanistic medicine. N Engl J Med 1983, 308:1059-1063.

10. Irby DM. Clinical teacher effectiveness in medicine. J Med Educ 1978, 53:808-815.

11. Irby DM. Clinical teaching and the clinical teacher. J Med Educ 1986, 61(Part 2):35-45.

12. Kassirer JP. Teaching clinical medicine by iterative hypothesis testing: let's preach what we practice. N Engl J Med 1983, 309:921-923.

13. Lana-Peixoto MA. A residência médica e o título de especialista em neurologia: a visăo da Academia Brasileira de Neurologia. Arq Neuropsiquiatr 1989, 47:503-505.

14. Lana-Peixoto MA. O neurologista e a literatura médica: o processo de educação interminável. Arq Neuropsiquiatr 1992, 50:244-252.

15. Martin JB. Training physician-scientists for the 1990s. Aclad Med 1991, 66:123-129.

16. Miller GE. The assessment of clinical skills/competence/performance. Acad Med 1990, 65(Suppl):63-67.

17. Miller S. Physicians for the twenty-first century: report of the project panel on the general professional education of the physician and college preparation for medicine. $\mathrm{J}$ Med Educ 1989, 59(Part 2):1-31.

18. Miller S. Emerging perspectives on the general professional education of the physician. $J$ Med Educ 1989, 59(Part 2):59-74.

19. Schimidt HG, Norman GR, Boshuizen HPA. A cognitive perspective of medical expertise: theory and implications. Acad Med 1990, 65:611-621.

20. Schroeder SA. Expanding the site of clinical education: moving beyond the hospital walls. J Gen Intern Med 1988, 3(Suppl):55-64.

21. Shapiro HT. Medical education and the university. J Med Educ 1986, 61:81-91.

21. Small PA. Consequences for medical education of problem-solving in science and medicine. J Med Educ 1988, 63:848-853.

23. Stemmler EJ. Medical education: is it? J Med Educ 1988, 63:81-91.

24. Weldon VV. Why the dinosaurs died: extinction or Evolution? J Med Educ 1987, 62: 109-115. 